OPEN ACCESS

Edited by:

Juan Bellas,

Spanish Institute of

Oceanography, Spain

Reviewed by:

Begoña Jiménez,

Spanish National Research Council

(CSIC), Spain

Pauline Pannetier

Heidelberg University, Germany

${ }^{*}$ Correspondence:

Christine Schönlau

christine.schonlau@oru.se

Specialty section:

This article was submitted to

Marine Pollution

a section of the journal

Frontiers in Environmental Science

Received: 15 February 2019

Accepted: 18 July 2019

Published: 02 August 2019

Citation:

Schönlau C, Larsson M, Dubocq F

Rotander $A$, van der Zande $R$,

Engwall M and Kärrman A (2019)

Effect-Directed Analysis of Ah

Receptor-Mediated Potencies in Microplastics Deployed in a Remote

Tropical Marine Environment.

Front. Environ. Sci. 7:120.

doi: 10.3389/fenvs.2019.00120

\section{Effect-Directed Analysis of Ah Receptor-Mediated Potencies in Microplastics Deployed in a Remote Tropical Marine Environment}

\author{
Christine Schönlau ${ }^{1 *}$, Maria Larsson ${ }^{1}$, Florian Dubocq ${ }^{1}$, Anna Rotander ${ }^{1}$, \\ Rene van der Zande ${ }^{2}$, Magnus Engwall ${ }^{1}$ and Anna Kärrman ${ }^{1}$ \\ ${ }^{1}$ MTM Research Centre, School of Science and Technology, Örebro University, Örebro, Sweden, ${ }^{2}$ Coral Reef Ecosystems \\ Lab and Global Change Institute, The University of Queensland, St. Lucia, QLD, Australia
}

To facilitate the study of potential harmful compounds sorbed to microplastics, an effect-directed analysis using the DR CALUX ${ }^{\circledR}$ assay as screening tool for Aryl hydrocarbon receptor (AhR)-active compounds in extracts of marine deployed microplastics and chemical analysis of hydrophobic organic compounds (HOCs) was conducted. Pellets of three plastic polymers [low-density polyethylene (LDPE), high-density polyethylene (HDPE) and high-impact polystyrene (HIPS)] were deployed at Heron Island in the Great Barrier Reef, Australia, for up to 8 months. Detected AhR-mediated potencies (bio-TEQs) of extracted plastic pellets ranged from 15 to 100 $\mathrm{pg} / \mathrm{g}$. Contributions of target HOCs to the overall bioactivities were negligible. To identify the major contributors, remaining plastic pellets were used for fractionation with a gas chromatography (GC) fractionation platform featuring parallel mass spectrometric (MS) detection. The bioassay analysis showed two bioactive fractions of each polymer with bio-TEQs ranging from 5.7 to $14 \mathrm{pg} / \mathrm{g}$. High resolution MS was used in order to identify bioactive compounds in the fractions. No AhR agonists could be identified in fractions of HDPE or LDPE. Via a multivariate statistical approach the polystyrene (PS) trimer 1e- Phenyl-4e-(1- phenylethyl)-tetralin was identified in fractions of HIPS and in fractions of the blank polymer of HIPS.

Keywords: polyethylene, polystyrene, PCBs, reporter gene assay, fractionation

\section{INTRODUCTION}

Hydrophobic organic compounds (HOCs) are able to sorb to plastics from the ambient environment, especially polyethylene (PE) has been recognized as a good sorbent for HOCs and has been utilized as a passive sampler in various studies (Zabiegala et al., 2010). Polycyclic aromatic hydrocarbons (PAHs), polychlorinated biphenyls (PCBs), pesticides, such as chlordanes, dichlorodiphenyltrichloroethane (DDT) and its breakdown products, and polybrominated diphenyl ethers (PBDEs) have been frequently detected on marine microplastics (Mato et al., 2001; Rios et al., 2007; Hirai et al., 2011; Van et al., 2012). Furthermore, plastics often contain additives to provide the end-products with the desired properties. One concern is that additives leach out from the plastics, as well as unreacted monomers or oligomers from the plastics themselves (Teuten et al., 2009). The importance of plastics as a vector for transferring HOCs to organisms is yet unclear, 
but has been debated by scientists in the past decade. In contrast to increasing the exposure to HOCs upon ingestion of microplastics, it has been hypothesized that ingested microplastics rather act as passive samplers for HOCs, therefore reflecting tissue concentrations of such compounds (Herzke et al., 2016) or can even act as a sink for pollutants, hence decreasing the bioaccumulation of pollutants (Koelmans et al., 2016). A better knowledge of identities and concentrations of sorbed HOCs in marine plastics would help in risk assessment of plastics. This knowledge can best be gained under field conditions in which microplastics are exposed to real life scenarios with stressors that microplastics have to face in the marine environment.

The Aryl hydrocarbon receptor (AhR) mediates toxic effects of several HOCs, including polychlorinated dibenzo-p-dioxins and furans (PCDD/Fs), coplanar non-di-ortho-substituted PCBs, and some PAHs (Denison and Heath-Pagliuso, 1998; Marlowe and Puga, 2005). In a previous study we assessed AhR-mediated potencies for the first time for extracts of plastic pellets of lowdensity polyethylene (LDPE), polypropylene (PP), polyethylene terephthalate (PET), and polyvinylchloride (PVC) (Schönlau et al., 2019). The samples in the previous study have been deployed in an urban marina which was highly impacted by anthropogenic activity. In some samples the potency could mainly be attributed to quantified PAHs, but in other samples the analyzed PAHs only contributed partially to the overall AhRmediated efficacies. Therefore, it is of interest to assess also the contribution of other AhR agonist, such as coplanar non-diortho-substituted PCBs and PCDD/Fs.

The challenge with environmental samples, including marine microplastics, is that they usually contain complex mixtures of chemicals and chemical analysis can often not detect all toxicologically relevant compounds. In effect-directed analysis (EDA), the complexity of a mixture is sequentially reduced by separating the components into different fractions. The potential toxicity of these fractions is thereafter tested in a relevant bioassay. Fractions that demonstrate great bioactivities are then subjected to chemical analysis to identify bioactive compounds, or can be further fractionated if the fraction is still too complex. In general, liquid chromatography (LC) is used for fractionation of samples in EDA studies (Lubcke-von Varel et al., 2008; Weiss et al., 2009; Grung et al., 2011), but gas chromatography (GC) is the preferred separation technique for analysis of non-polar organic pollutants in environmental samples (Poster et al., 2006; Tombesi et al., 2017; Bjurlid et al., 2018). In recent years, a new GC-based fractionation platform with a post-column introduction of a trap solvent for fraction collection has been developed (Pieke et al., 2013; Jonker et al., 2016, 2017). This platform allows for fractionation during the entire time of a GC run, thus covering a complete chromatogram. Recently, the GC fractionation platform has been coupled to mass spectrometric (MS) detection for parallel analysis of compounds, and was successfully evaluated for the fractionation of a mixture of nalkanes $\left(\mathrm{C}_{7}-\mathrm{C}_{30}\right)$, and a mixture of two pesticides, vinclozolin and p,p'-DDE (Jonker et al., 2017).

The aim of this study was to assess the levels of AhR-mediated potencies in extracts of three types of mass produced pristine plastic pellets deployed in a remote site and to estimate the contribution of sorbed HOCs to the overall biological effect. Additionally, for the first time a GC-based fractionation platform was utilized for the identification of HOCs sorbed to marine microplastics and tested for its suitability. Pristine plastic pellets, which are plastic pellets that are usually used as raw material for manufacturing of plastic products [LDPE, high-density polyethylene (HDPE) and high-impact polystyrene (HIPS)], were deployed at Heron Island in the Great Barrier Reef, Australia, and were analyzed for their potential to elicit an AhR- mediated activity in the DR CALUX ${ }^{\circledR}$ (Dioxin Receptor Chemical Activated LUciferase gene eXpression) assay. In order to identify the compounds causing the AhR-mediated activity a two-step approach was conducted. Firstly potency balance calculations were conducted, comparing predicted activities of the target HOCs, including PCBs, PBDEs, pesticides, and PCDD/Fs, in the microplastics to the observed activities in the DR-CALUX ${ }^{\circledR}$ bioassay. If the observed bioassay activities were only partially explained, the second step was GC-MS fractionation followed by bioassay analysis and chemical analysis of the bioactive fractions using high resolution GC-Orbitrap ${ }^{\mathrm{TM}} \mathrm{MS}$.

\section{MATERIALS AND METHODS}

\section{Chemicals}

SupraSolv ${ }^{\circledR}$ n-hexane ( $\geq 98 \%$ ) (Merck, Darmstadt, Germany) was used for the extractions and dimethyl sulfoxide (DMSO) (99.9\%) (Sigma Aldrich, Stockholm, Sweden) was used for dissolving extracts in bioassay test medium. Toluene $(>99.5)$ and Heptane $(\geq 99 \%)$, were used for fraction collection (Honeywell, Riedel-de Haën, Steinheim, Germany). Tetradecane for chemical analysis was purchased from Sigma Aldrich (Stockholm, Sweden).

The bioassay reagent Steady Lite plus $^{\mathrm{TM}}$ was purchased from Perkin Elmer (Hägersten, Sweden). A 2,3,7,8tetrachlorodibenzo- $p$-dioxin (TCDD) standard, with a purity of 99.1\%, was from AccuStandard Inc. (New Haven, USA).

Target chemicals included PCBs, PBDEs, pesticides and PCDD/Fs. Target chemicals after fractionation also included PAHs. Information regarding native and labeled chemical standards are presented in the Supplementary Material.

\section{Deployment of Plastics}

Three pristine plastic pellets, LDPE, HDPE and HIPS were deployed on the 14th of February 2015 for 8 months at Heron Island, Great Barrier Reef, Australia (For information about coordinates and sampling dates see Table S1 of the Supplementary Material). HDPE samples were spherical with a diameter of $3 \mathrm{~mm}$, HIPS and LDPE samples were cylindrical with a length of $4 \mathrm{~mm}$ and a height of $2-3 \mathrm{~mm}$. HDPE granules contained an unknown UV stabilizer. The samples were deployed in two stainless steel cages which contained larger mesh bags (10 $\mathrm{mm}$ mesh size) to separate each plastic polymer from another (Figure S1). The larger mesh bags contained smaller mesh bags (2-3 mm mesh size) with approximately $4 \mathrm{~g}$ of a polymer for every sampling time point. The metal cages were anchored in an open sandy patch, between coral rubble in a shallow part of 
Heron Island lagoon, using sand pegs. At low tide the water depth at the site was approximately $0.5 \mathrm{~m}$, and between 1.5 and $2.5 \mathrm{~m}$ depth at high tide, depending on the weather conditions. In order to avoid too much sand accumulation on the samples and secure them against tidal movements, two layers of a black knitted shade cloth were used to cover the samples. The shade cloth was cleaned once a month from sand. Samples were taken after 1, 3, 6, and 8 months of deployment, wrapped in alumina foil and stored at $-20^{\circ} \mathrm{C}$ until analysis. For all LDPE samples replicates were available, one from each cage. For HDPE and HIPS a replicate sample was only available for the first month of deployment, after that only one sample of each polymer was collected from the first cage.

\section{Extraction of Microplastics}

Prior to extraction of real samples, pellets of LDPE and HDPE were spiked with labeled PCBs, pesticides, OCDD and PBDE\#47 standards to test the extraction efficiency of the compounds (Table S2). Samples were rinsed with ultrapure water to remove sand and bigger particles, and left to dry in the fume hood. Approximately $3 \mathrm{~g}$ of plastic pellets were extracted with $4 \mathrm{~mL}$ of $\mathrm{n}$-hexane by ultrasonication for $60 \mathrm{~min}$. The extracts were transferred to brown glass vials and extraction was repeated with $3 \mathrm{~mL}$ of $\mathrm{n}$-hexane. Both extracts were pooled together and $1-2 \mathrm{~mL}$ of concentrated sulfuric acid (98\%) was added to the extracts to eliminate organic matter. Non-persistent organic chemicals were also degraded in this step. After vortex mixing, and incubation at room temperature for $3 \mathrm{~h}$ and centrifugation at 4,500 rpm for $10 \mathrm{~min}$, the supernatant was transferred to brown glass vials. Finally, $2 \mathrm{~mL}$ of $\mathrm{n}$-hexane were pipetted into the centrifuge vials, vortexed and centrifuged at 4,500 rpm for $10 \mathrm{~min}$. The remaining supernatant was transferred to the brown glass vials. Extracts were evaporated under a gentle nitrogen flow to $1 \mathrm{~mL}$ of extract. Subsequently extracts were split into two aliquots. One aliquot was solvent exchanged to DMSO for bioassay testing. The other aliquot was spiked with internal standard (IS) and recovery standard (RS), solvent exchanged to tetradecane and evaporated under a gentle nitrogen flow to a volume of 25 $\mu \mathrm{L}$ for chemical analysis. Additionally to the deployed samples, pristine plastic pellets of each polymer type were extracted as polymer blanks. The samples are named hereafter by their polymer acronym, LDPE, HDPE and HIPS, the cage number written as I or II, and the time period of deployment written as $1,3,6$, or $8 \mathrm{~m}$.

In order to decrease detection limits for the fractionation of real samples, the remaining $1 \mathrm{~g}$ of each of the plastics collected at $1,3,6$, and 8 months of deployment and available replicates were pooled according to the type of polymer, resulting in 3 samples. Hereafter the pooled samples are named by the polymer acronym and the time span of deployment from 1 to 8 months written as 1-8 $\mathrm{m}$. For LDPE 1-8 $\mathrm{m} 8.56 \mathrm{~g}$, for HDPE 1-8 $\mathrm{m} 5.04 \mathrm{~g}$ and for HIPS 1-8 m $5.56 \mathrm{~g}$ were extracted. Besides two process blanks without polymers, $10 \mathrm{~g}$ of each plastic pellet were extracted as polymer blank samples. The extraction procedure was the same as for the individual, non-pooled samples with the exception that $20 \mathrm{~mL}$ of extraction solvent was used and the obtained extracts were evaporated to a volume of $5-6 \mathrm{~mL}$ in a rotary evaporator. All extracts were split into two aliquots, one aliquot was solvent exchanged to DMSO for bioassay testing and the second aliquot was solvent exchanged to toluene and evaporated to a volume of $300 \mu \mathrm{L}$ for further fractionation.

\section{Chemical Analysis}

For analysis of target compounds in individual plastic samples an Agilent 7890 A GC system (Agilent Technologies, Santa Clara, USA) coupled to atmospheric pressure chemical ionization tandem quadrupole mass spectrometer Xevo TQ-S (APCIMS/MS), (Waters Corporation, Milford, USA), was used. GC separation was achieved using a $30 \mathrm{~m} \times 0.25 \mathrm{~mm}, 0.25 \mu \mathrm{m}$ film thickness, DB-5MS capillary column ( $\&$ W Agilent Technologies, Santa Clara, USA). All samples were injected at $280^{\circ} \mathrm{C}$ in splitless mode. Measurements were done in multiple reaction mode (MRM).

Quantification of target compounds in individual plastic samples was done by use of the software MassLynx V4.1 (Waters Cooperation, Milford, USA). Target compounds were quantified by use of a one-point calibration and a quantification standard containing target compounds. The quantification standard was analyzed in the beginning and in the end of a run. The limit of detection (LOD) was defined as the concentration of the native compounds in the quantification standard divided by three times the signal-to-noise ratio, and the limit of quantification (LOQ) was defined as the concentration of the compound divided by ten times the signal-to-noise ratio.

A GC fractionation platform with parallel MS detection, as described by Jonker et al. (2017), was used for fractionation of pooled plastic samples. Prior to fractionation of real samples, the platform was evaluated for its performance by injection and qualitative analysis of five individual standards, and a mixture of 134 known compounds, including PAHs (5 ng/ $\mu \mathrm{L})$, PCBs (20-40 pg/ $\mathrm{LL})$, PCDD/Fs $(20-100 \mathrm{pg} / \mu \mathrm{L})$, PBDEs $(20$ $\mathrm{pg} / \mu \mathrm{L})$, and pesticides $(20 \mathrm{pg} / \mu \mathrm{L})$. The GC fractionation platform consisted of an Agilent 7890 B gas chromatograph coupled to a 5977 B series low-resolution mass spectrometer (GC/LRMS) and a multipurpose sampler (GERSTEL GmbH \& Co. KG, Mühlheim an der Ruhr, Germany). An Agilent 1260 Infinity II LC pump was connected to an inert twoway splitter (Agilent Technologies, Santa Clara, USA) that allowed the post-GC column split. One part $(\sim 1 \%)$ of the eluate was guided toward the MS via a $1 \mathrm{~m} \times 0.1 \mathrm{~mm}$ internal diameter, deactivated fused silica column (Agilent Technologies). The other part was guided toward fraction collection via a $1.2 \mathrm{~m} \times 0.32 \mathrm{~mm}$ internal diameter deactivated fused silica column. The instrument components were controlled by the Agilent Masshunter software. The multipurpose sampler, used for injection and fraction collection, was controlled by the DVLS GC fractionation software, version 1.0.2.6. (Da Vinci Laboratory Solutions, Rotterdam, The Netherlands). Separation of compounds was achieved on a capillary column $(30 \mathrm{~m}$ $\times 0.25 \mathrm{~mm}, 0.15 \mu \mathrm{m}$ film thickness) (Select PAH; Agilent Technologies). Measurements of the mixture and real extracts were performed in full scan mode from $\mathrm{m} / \mathrm{z} 50$ to 550 . Measurements of single native standards were done in selected 
ion monitoring mode (SIM). Seventeen fractions were collected between 4 and $54 \mathrm{~min}$ with 15 consecutive injections per sample. It was fractionated with 60 s/well into glass inserts. Fractions of three sequential inserts were pooled into $1.5 \mathrm{~mL}$ brown glass vials, which resulted in 17 fractions. The last fraction, fraction 17, consisted of a pool of two inserts. For each sample two batches of 17 fractions were obtained, one batch was subjected to bioassay analysis and the second batch was used for further targeted and non-targeted chemical analysis.

Qualitative chemical analysis of the obtained fractions was performed on a ThermoFisher Scientific Q Exactive ${ }^{\mathrm{TM}}$ Quadrupole-Orbitrap ${ }^{\mathrm{TM}}$ Mass Spectrometer at a resolution of 60,000 in full scan mode with a scan range of $\mathrm{m} / \mathrm{z} 53.4$ to 800 and a simultaneous SIM mode, for 49 selected $\mathrm{m} / \mathrm{z}$ (Table S3). Suspect screening for additives, which are commonly used in the specific plastic polymers, was done on blank and deployed plastic samples (Table S4). XCMS online (The Scripps Research Institute, La Jolla, USA) was used to preprocess (peak detection, filtering and alignment) and post process (ANOVA statistical test) raw datasets. Graphical tools resulting from the statistical test as principal component analysis (PCA) were used to detect outliers. In addition, mass spectra of the most abundant signals were extracted from the chromatogram and formulae and structures were tentatively determined using library searches including National Institute for Standards and Technology (NIST) library or by literature search. All the $\mathrm{m} / \mathrm{z}$ extracted from the nontarget analysis were below two parts- per-million ( $\mathrm{ppm}$ ) accuracy compared with the theoretical $\mathrm{m} / \mathrm{z}$.

For more details regarding the used GC temperature programs on each instrument refer to the Supplementary Material.

\section{DR CALUX ${ }^{\circledR}$ Assay}

AhR-mediated potencies were measured by use of the DR CALUX ${ }^{\circledR}$ assay, using a recombinant rat hepatoma cell line with a stably transfected luciferase reporter gene (H4IIepGudluc1.1cells) (Murk et al., 1996). The cell line was obtained from BioDetection Systems (BDS) (Amsterdam, The Netherlands). The DR CALUX ${ }^{\circledR}$ assay was performed as previously described for the H4IIE-luc assay in Larsson et al. (2013). Extracts of individual plastic samples were tested for $24 \mathrm{~h}$ and $72 \mathrm{~h}$ exposure. Sample extracts were prepared as 3-fold serial dilutions in culture medium and added to the test plates in six different test concentrations. Dilutions of fractionated samples were prepared as 4-fold serial dilutions. Cells were microscopically examined for cytotoxicity before and after exposure. Luciferase activity in each well was measured in a luminometer (Fluostar, Omega). Concentration-response curves for TCDD and extracts were obtained by use of a sigmoidal concentration-response (variable slope) equation (GraphPad Prism ${ }^{\circledR} 5.0$ software). All individual plastic samples were tested in three independent experiments. Bioactive fractions were tested twice.

\section{Data Analysis of Bioassay Results}

Bioassay derived TCDD equivalents (bio-TEQs), were calculated from concentration-response curves by relating the luciferase induction potencies of the samples to that of the TCDD standard as described in Larsson et al. (2013). Samples that had a TCDD $\mathrm{EC}_{50}$ value between 6 and $18 \mathrm{pM}$ and a maximal induction factor (MIF) between 7 and 17 were used in TEQ calculations. Limit of detection (LOD) in the assay is the mean of the solvent control (DMSO) plus three times the standard deviation.

Chemically derived TCDD equivalents (chem-TEQs) were calculated as the sum of the product of individual concentrations of PCBs and PCDD/Fs multiplied with their H4IIE-luc or CALUX assay specific relative potency factors (REP) based on $\mathrm{EC}_{50}$ or $\mathrm{EC}_{20-80}$ values. The applied REP values were taken from Lee et al. (2013) and USEPA (2014). Potency balance calculations between bio- TEQs and chem-TEQs were conducted to determine the contribution of analyzed chemicals to the total induced AhR-mediated potency.

Statistical analysis was done by using GraphPad Prism ${ }^{\circledR} 5.0$ software and the significance level was set to $p<0.05$.

\section{RESULTS}

\section{AhR-Mediated Potencies of Deployed Plastics Over Time}

A total of 21 samples were examined for their AhR-mediated potency, including three blank samples of each polymer. In the $24 \mathrm{~h}$ assay, most of the samples had responses $<50 \%$ of the TCDD maximum induction (TMI). Bio-TEQs for LDPE and HDPE samples were calculated at the $25 \%$ effect level $\left(\mathrm{EC}_{25}\right)$ of TMI (Figure 1) (Villeneuve et al., 2000). Cytotoxicity was observed for all deployed HIPS samples at the greatest concentration tested in the assay. For some replicates also the second greatest concentration tested showed cytotoxicity. Therefore, bio-TEQs for HIPS samples were calculated based on the $\mathrm{EC}_{20}$ level of TMI, where no cytotoxicity was observed. Among the three polymers HDPE showed the greatest AhR-mediated potency with a bioTEQ of $100 \mathrm{pg} / \mathrm{g}$ after 8 months of deployment, followed by HIPS with $69 \mathrm{pg} / \mathrm{g}\left(\mathrm{EC}_{20}\right)$, and LDPE with $57 \mathrm{pg} / \mathrm{g}$ (LDPE I $8 \mathrm{~m}$ ) and 41 $\mathrm{pg} / \mathrm{g}$ (LDPE II $8 \mathrm{~m}$ ), respectively (Table S6). There was, however, no significant difference among the three polymers (ANOVA, $p=$ $0.53)$, while there were significant differences in the deployment times for $\operatorname{HDPE}(p<0.0001)$, and $\operatorname{LDPE}(p=0.001)$. The polymer blank of HDPE had an AhR-mediated activity with a bio-TEQ of $7.6 \mathrm{pg} / \mathrm{g}\left(\mathrm{EC}_{20}\right)$. Polymer blanks of LDPE and HIPS did not induce measurable AhR-mediated activities. The blank sample of HIPS was observed to be cytotoxic in the highest concentration tested in the bioassay.

In order to assess if the AhR-mediated potencies observed in the $24 \mathrm{~h}$ assay were due to compounds resistant to cellular metabolism, like PCDD/Fs, the AhR-mediated potencies of the extracts of plastic samples were also investigated following $72 \mathrm{~h}$ of exposure. None of the samples induced measurable AhRmediated potencies after an exposure time of $72 \mathrm{~h}$.

\section{Occurrence of HOCs on Deployed Plastics}

The chemical analysis showed low concentrations of target HOCs, which exceeded polymer blank concentrations in a few samples. All polymer blanks contained measurable levels of PCBs ranging from $84 \mathrm{pg} / \mathrm{g}$ in HIPS to $355 \mathrm{pg} / \mathrm{g}$ in HDPE (Table S7). HDPE samples demonstrated the greatest 


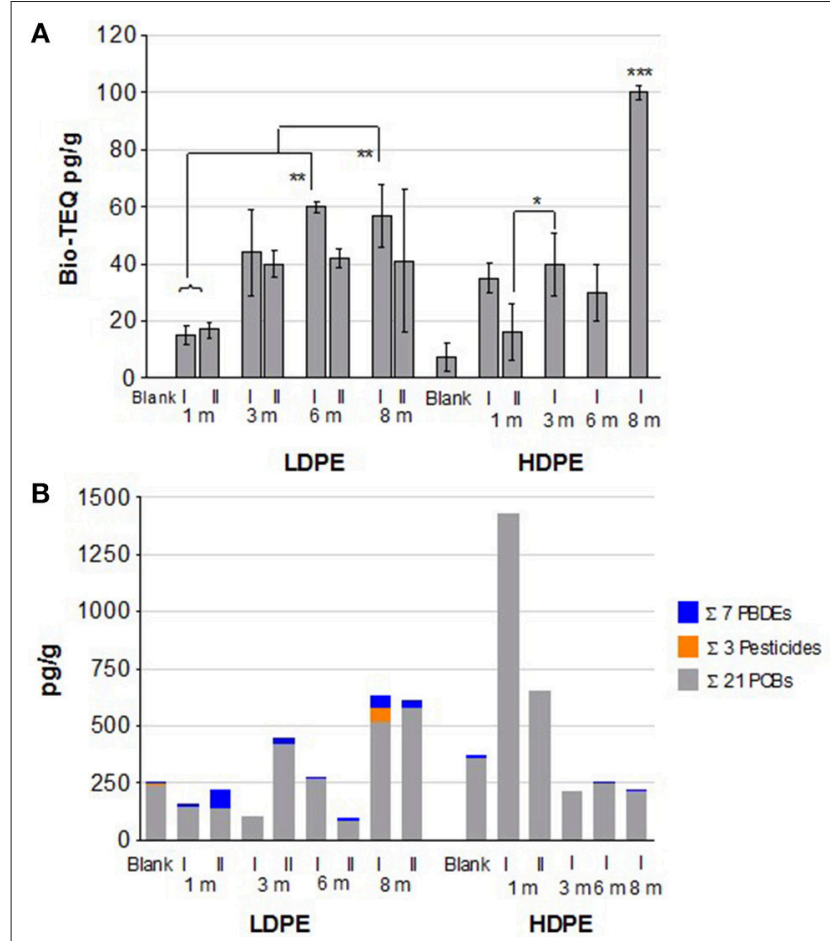

FIGURE 1 | Bioassay (DR CALUX ${ }^{\circledR}$ ) and chemical analysis of extracts of deployed LDPE and HDPE pellets. (A) Bio-TEQs $\left(\mathrm{EC}_{25}\right)$ in $\mathrm{pg} / \mathrm{g}$ calculated following a $24 \mathrm{~h}$ exposure given as a mean of $\mathrm{n}$ replicates $(n=3-4)$ and the standard deviation as error bars. Significance levels of ANOVA followed by Tuckey's post hoc test are indicated as ${ }^{\star} p<0.01 ;{ }^{\star \star} p<0.001$; ${ }^{* \star *} p<0.0001$. (B) Sum of concentrations of detectable HOCs above LOQ in pg/g in extracts of plastic pellets. PBDEs included congeners $28,47,85,99,100,154$, and 183; pesticides included heptachlor, t-chlordane, and p, p'-DDD; PCBs included congeners 44, 49, 52, 66, 70, 74, 79, 87, 95, 99, 101, 105, 110 , $118,138,149,153,155,156,157$, and 162.

total PCB concentrations of $1,430 \mathrm{pg} / \mathrm{g}$ and $652 \mathrm{pg} / \mathrm{g}$ after 1 month of deployment, followed by a decrease of concentrations in the following sampling months (Figure 1). LDPE samples demonstrated an increase in total PCB concentrations from a polymer blank concentration of $239 \mathrm{pg} / \mathrm{g}$ to $519 \mathrm{pg} / \mathrm{g}$ and 577 $\mathrm{pg} / \mathrm{g}$ after 8 months of deployment. The quantification of HOCs in HIPS samples with a one-dimensional GC was not always possible due to the complex matrix of this polymer, which also has been noticed in another study (Rochman et al., 2013b). Therefore, the results for HIPS were excluded from the data set. While in the blank polymer of LDPE PCB \#81 had the greatest concentration among detected PCBs (81 pg/g), PCB \#153 and \#138 had the greatest concentrations in blank polymer of HDPE (155 pg/g and $111 \mathrm{pg} / \mathrm{g}$, respectively). In the deployed LDPE pellets PCB \#138 and \#153 had the greatest concentrations among detected PCBs, except for the sample LDPE II $1 \mathrm{~m}$ in which PCB \#49 had the greatest concentration among PCBs. Also in deployed samples of HDPE the PCB congeners \#138 and \#153 were the major components among detected PCBs. The seven target PBDEs \#28, \#47, \#85, \#99, \#100, \#154, \#183 were detected in different deployed LDPE and HDPE samples. BDE
\#47 had the greatest concentrations among detected PBDEs in all samples, including blank polymers of LDPE and HDPE, except for LDPE I $3 \mathrm{~m}$ in which PBDE \#28 was most abundant. PBDE \#28 was detected in all deployed samples and blank HDPE as well, although the concentration in HDPE I $1 \mathrm{~m}$ was below the LOQ (Table S7). Among the three detected pesticides, heptachlor, p, p'-DDD, and t-chlordane, only heptachlor had a concentration above the LOQ in sample LDPE I $8 \mathrm{~m}$, and p, p'-DDD in the polymer blank of LDPE.

\section{Potency Balance Calculations}

Chem-TEQ values were calculated based on the analyzed PCBs and $\mathrm{PCDD} / \mathrm{Fs}$ to assess the contribution of these compounds to the overall bioassay activities. Among those two compound classes only the known AhR agonists PCB 105, 118, 156, 157, and OCDD had detectable concentrations in some of the samples. The contribution of these five compounds to the measured bioTEQs was negligible, calculated chem-TEQs could only explain $1.3 \times 10^{-6} \%$ (LDPE I $6 \mathrm{~m}$ ) to $0.001 \%$ (HDPE II $1 \mathrm{~m}$ ) (Table S6). Also concentrations which were below LOQ but above LOD levels were considered for the calculation of chem-TEQs.

\section{Effect-Directed Analysis of Pooled Plastic Extracts \\ Performance of the GC-MS Fractionation Platform}

To further investigate and identify the AhR-active compounds in deployed plastic pellets a GC-MS fractionation platform was used to collect 17 fractions of each polymer extract. As a proof of principle, the fractionation performance was evaluated by repeated injections, and collecting fractions, of a known mixture of 134 compounds containing PAHs, PCBs, PBDEs, PCDD/Fs and pesticides. The consistency of retention times was proven to be good, $<0.1$ min variation.

Most compounds of the mixture, except for some pesticides (Aldrin, dieldrin, methoxychlor, isodrin, heptachlor, endrin, heptachlor exo- and endo epoxide, alpha and beta endosulfan, oxy chlordane), PCB \#111, and four PBDEs (\#99, \#85, \#138, and \#183), were detectable in collected fractions (Table S7). Recoveries of the 118 detected compounds were calculated using theoretical concentrations and the quantified concentrations in fractions by analysis with GC-HRMS. The recoveries of the detected compounds, excluding the $16 \mathrm{PAHs}$, ranged from 4 $\%$ (hexachlorobenzene) to $85 \%$ (PCB \#138, and \#153) and recoveries were $<50 \%$ for 96 out of 102 compounds. A total of 41 compounds were close to instrumental detection limits. Recoveries for the low molecular weight PAHs were above theoretical concentrations (144-455\%), while the high molecular weight PAHs benzo[a]anthracene, benzo[b]fluoranthene, benzo[a]pyrene, benzo[g,h,i]perylene and dibenz[a,h] anthracene had acceptable recoveries between 85 and 106\%.

\section{AhR-Mediated Activities}

In order to increase concentrations of AhR agonists present in the samples, plastic pellets from the different time points, 1 to 8 months, were pooled according to polymer type and 17 fractions were collected of 15 consecutive injections. AhRmediated responses were observed in fractions 12 and 14 of 


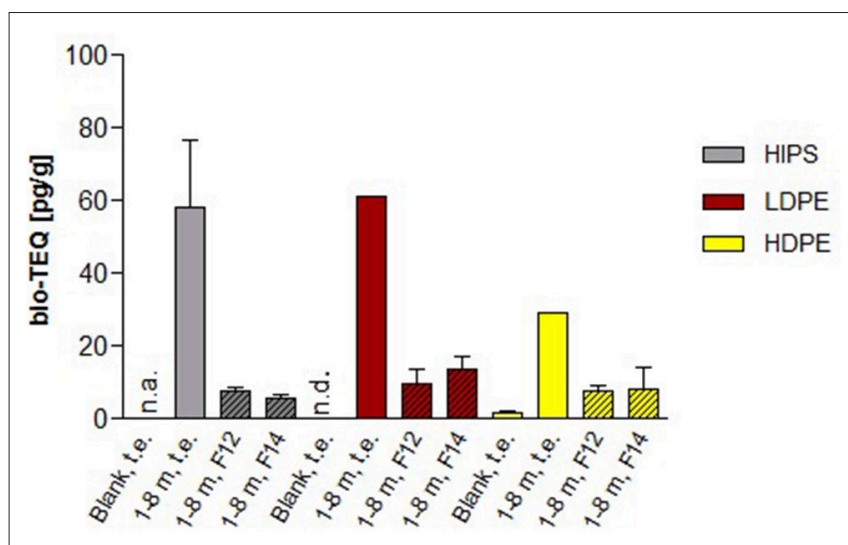

FIGURE 2 | Overview of calculated bio-TEQs $\left(\mathrm{EC}_{10}\right)$ in pg/g in total and fractionated extracts of pooled plastics. n.a. = not analyzed; n.d. = AhR-mediated potency less than LOD in the assay; t.e. = total extract, unfractionated.

all deployed polymers. Their maximum responses were $<20 \%$ of TMI for most of the fractions, therefore bio-TEQs were calculated based on $\mathrm{EC}_{10}$. Only fraction 14 of LDPE $1-8 \mathrm{~m}$ induced a response up to $20 \%$ of TMI. Calculated bio-TEQs ranged from $5.7 \mathrm{pg} / \mathrm{g}$ in fraction 14 of HIPS $1-8 \mathrm{~m}$ to $14 \mathrm{pg} / \mathrm{g}$ in fraction 14 of LDPE $1-8 \mathrm{~m}$. In fraction 12 bio-TEQs ranged from $7.6 \mathrm{pg} / \mathrm{g}$ (HIPS $1-8 \mathrm{~m}$ ) to $9.8 \mathrm{pg} / \mathrm{g}$ (LDPE $1-8 \mathrm{~m}$ ). Fraction $12 \mathrm{of}$ the HIPS blank showed a bioactivity with a bio-TEQ of $4.5 \mathrm{pg} / \mathrm{g}$, while fractionated polymer blanks of LDPE and HDPE did not induce measurable AhR-mediated activities. Fraction 11 of HIPS 1-8 $\mathrm{m}$ was observed to be cytotoxic. However, fraction 11 of the HIPS blank showed also cytotoxicity.

Additionally, non-fractionated total extracts of pooled pellets were examined for AhR-mediated potencies. Total extracts had greater bioactivities than individual fractions (Figure 2). Total extracts of HDPE and LDPE induced AhR-mediated responses up to $25 \%$ of TMI. Bio-TEQs $\left(\mathrm{EC}_{10}\right)$ for total extracts were 29 $\mathrm{pg} / \mathrm{g}$ in HDPE $1-8 \mathrm{~m}, 58 \mathrm{pg} / \mathrm{g}$ in HIPS $1-8 \mathrm{~m}$, and $61 \mathrm{pg} / \mathrm{g}$ in LDPE 1-8 m. Total extracts of polymer blanks were tested as well, except for HIPS. The total extract of blank HDPE showed an AhR-mediated potency of $1.6 \mathrm{pg} / \mathrm{g}$, whereas the potency of blank LDPE was less than the LOD in the assay. Although the total blank extract of HIPS was not tested in this batch of samples, a HIPS blank was tested in the analysis of individual samples of each time point of deployment. A cytotoxicity was observed before in the HIPS blank and was assumed to also occur in the second HIPS blank since the extraction procedure was the same and only the amount of extracted plastic pellets was higher.

\section{Chemical Analysis}

The compounds were fractionated according to their octanolwater partition coefficients (log $\mathrm{K}_{\mathrm{OW}}$ ) with more hydrophobic compounds in later eluting fractions. The cytotoxic fraction 11 of HIPS 1-8 $\mathrm{m}$ corresponded to the retention time of 34.12$37.12 \mathrm{~min}$ and comparison with fraction 11 of the standard mixture (see Table S7) showed that compounds with a log $\mathrm{K}_{\mathrm{OW}}$ between 6.73 (BDE 66) and 8 (PCB 208) were eluted in this fraction. The bioactive fractions 12 and 14 corresponded to the retention times of $37.12-40.12 \mathrm{~min}$ and $43.12-46.12 \mathrm{~min}$, respectively. Fraction 12 corresponded to compounds with a $\log \mathrm{K}_{\mathrm{OW}}$ between 6.92 (2,3,4,6,7,8-HxCDF) and 8.18 (PCB 209). Fraction 14 corresponded to compounds with a log $\mathrm{K}_{\mathrm{OW}}$ between 5.97 (benzo[a]pyrene) and 8.2 (OCDD).

As last part of the conducted effect-directed analysis in this study (Figure 3), bioactive fractions of deployed plastic pellets, the non-fractionated total extracts, and corresponding blank polymer fractions were subjected to a qualitative target, suspect, and non-target analysis (Tables S3-S5). Neighboring fractions of fractions 12 and 14 were analyzed as well.

The target analysis revealed naphthalene, fluorene, phenanthrene, anthracene, pyrene, and fluoranthene in different fractions of HIPS $1-8 \mathrm{~m}$ and blank HIPS. The intensities of detected PAHs were observed to be similar in blank HIPS compared to deployed HIPS, except for fluorene which was 2 orders of magnitude higher in the deployed sample compared to the blank polymer. The suspect screening of known plastic additives (Table S4), did not result in any detected compounds in blank or deployed plastic samples.

In order to identify unknown bioactive compounds multivariate statistics, PCA, were used on full scan MS data for both bioactive and non-bioactive fractions. Fraction 11 of HIPS $1-8 \mathrm{~m}$ and blank HIPS were segregated from other HIPS fractions in the score plot (Figure S2). In fraction $11 \mathrm{~m} / \mathrm{z}$ 129.1 with the retention time of $23.6 \mathrm{~min}$ (HIPS $1-8 \mathrm{~m}$ ) and $24.8 \mathrm{~min}$ (blank HIPS), and $\mathrm{m} / \mathrm{z} 91.1$ with the retention time of $23.7 \mathrm{~min}$ (HIPS 1-8 $\mathrm{m}$ ) and $24.6 \mathrm{~min}$ (blank HIPS) were found to be predominant ions. No difference among fractions of HDPE or among fractions of LDPE was observed in the score plot.

Additional spectral analysis of the major peaks ( $>10 \%$ of base peak) was performed (Table 1). In fraction 9 to 15 of HIPS $1-8 \mathrm{~m}$ and blank HIPS the peak at a retention time of $23.63 \mathrm{~min}$ was tentatively identified as 1e-Phenyl-4e-(1phenylethyl)-tetralin (accurate mass 312.1878, error $0 \mathrm{ppm}$ ) (Figure 4). The abundance in fraction 11 was one or two orders of magnitude higher than in the other fractions.

\section{DISCUSSION}

\section{AhR-Mediated Potencies of Deployed Plastics}

All extracts of deployed plastic pellets induced AhR-mediated potencies, but replicate samples of LDPE had no significantly increasing AhR-mediated potencies during time of deployment. However, a sorption of AhR agonists onto deployed plastic pellets was observed compared to polymer blank samples. The blank polymer of HDPE demonstrated a weak AhR-mediated potency as well, if the activity was due to contained additives could not be verified by chemical analysis. AhR-mediated potencies were only observed following a $24 \mathrm{~h}$ exposure to extracts of deployed plastic pellets. This indicates that AhR agonists, present in the samples, were degraded after $72 \mathrm{~h}$ due to cellular metabolism in the assay. Previous studies have demonstrated that more metabolic persistent chemicals, like PCDD/Fs and coplanar PCBs, are stable 


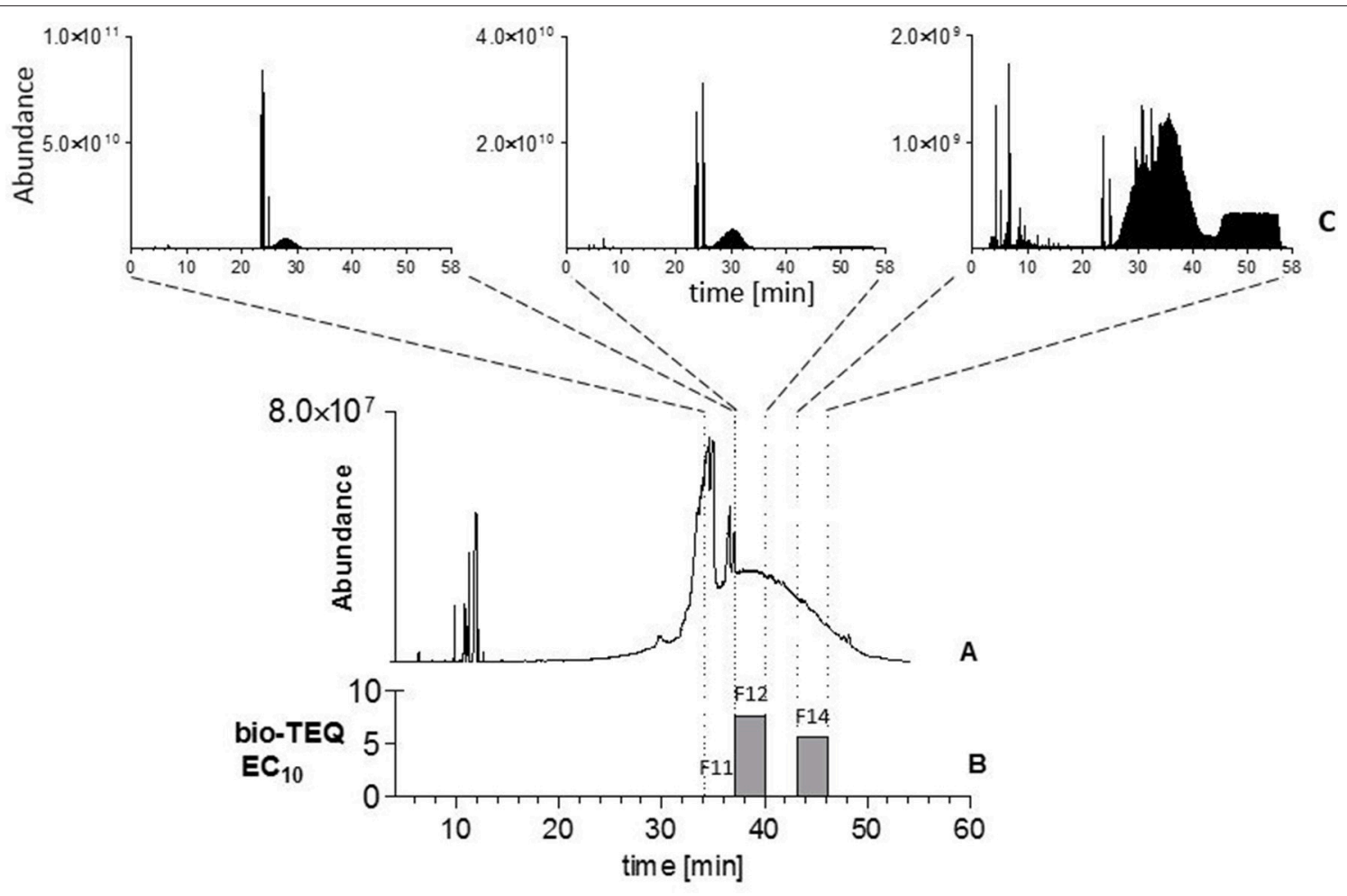

FIGURE 3 | Effect-directed analysis conducted in pooled plastic pellets of marine deployed HIPS. (A) GC- MS chromatogram of the pooled HIPS sample. (B) Measured bio-TEQs in pg/g (EC 10 ) of 17 fractions of pooled HIPS. Dashed lines represent fractions 11, 12, and 14, which induced AhR-mediated potencies in the DR CALUX $^{\circledR}$ (fraction 11 was cytotoxic). (C) GC-MS chromatograms of fractions 11, 12, and 14 of pooled HIPS from left to right analyzed with HRMS. Y-axis represents the abundance of detected peaks.
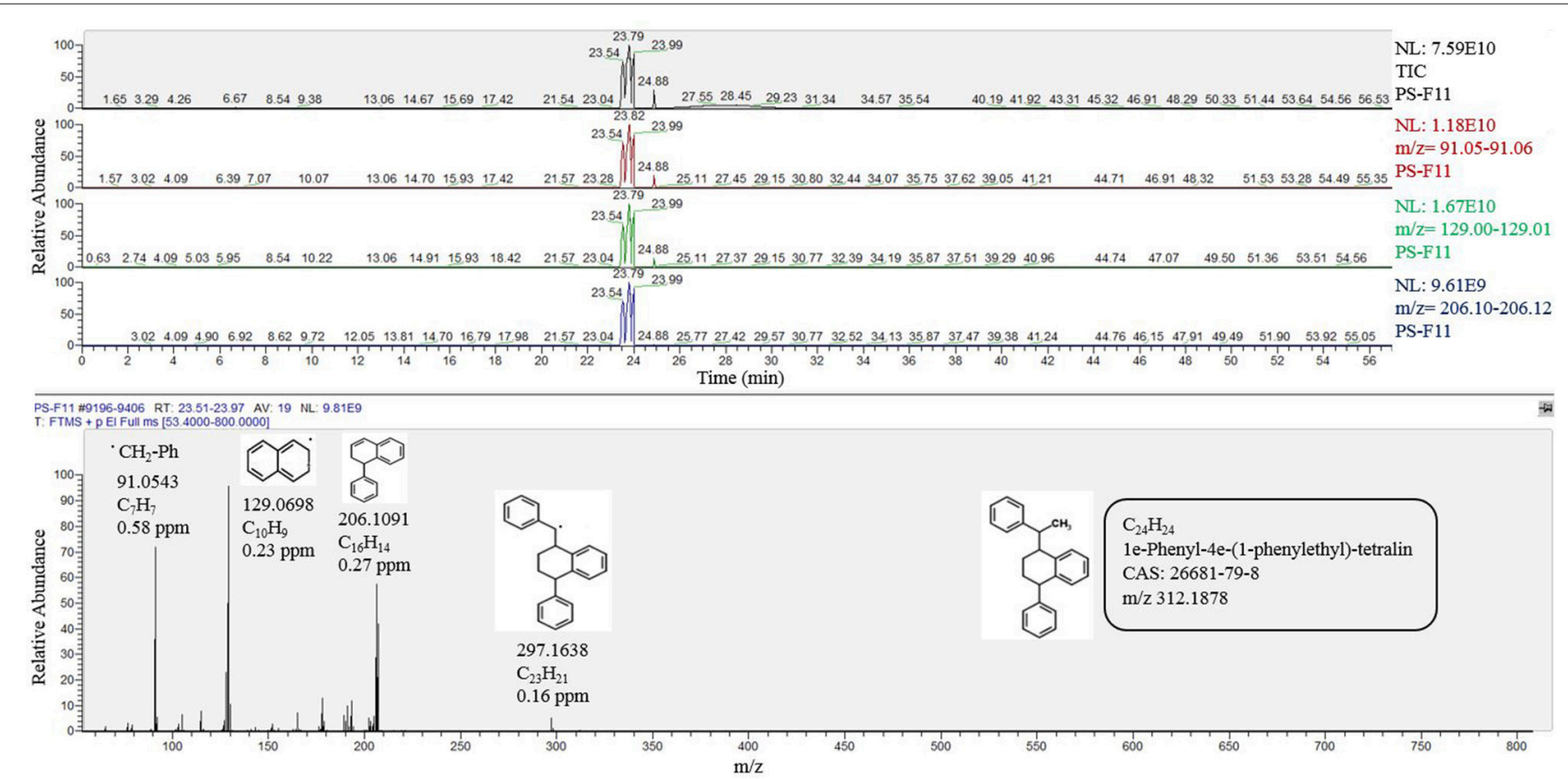

FIGURE 4 | Total ion chromatogram (TIC) and extracted masses of fragment ions of 1e- Phenyl- 4e- (1- phenylethyl)-tetralin (top) and mass spectra for 1e- Phenyl4e- (1- phenylethyl)- tetralin (bottom) in HIPS fraction 11. 
TABLE 1 | Detected major peaks (>10\% base peak) in the total ion chromatograms ( $\mathrm{m} / \mathrm{z}$ 53.4-800) of fractions of deployed and blank plastic polymers detected by GC-HRMS.

\begin{tabular}{|c|c|c|c|c|c|c|}
\hline Sample & Retention time [min] & $\mathrm{m} / \mathrm{z}$ & Formula & Mass error (ppm) & Molecular ion & $\begin{array}{c}\text { Identification } \\
\text { confidence } \\
\text { degree }^{\star}\end{array}$ \\
\hline $\begin{array}{l}\text { F 1-17 HIPS 1-8 m; F 1-17 } \\
\text { blank HIPS }\end{array}$ & 6.39 & $\begin{array}{l}162.1403 \\
148.1246 \\
133.1012 \\
105.0699\end{array}$ & $\begin{array}{c}\mathrm{C}_{12} \mathrm{H}_{18} \\
\mathrm{C}_{11} \mathrm{H}_{16} \\
\mathrm{C}_{10} \mathrm{H}_{13} \\
\mathrm{C}_{8} \mathrm{H}_{9}\end{array}$ & $\begin{array}{c}-0.02 \\
-0.00 \\
0.01 \\
0.06\end{array}$ & Hexamethylben-zene & $2 a)$ \\
\hline $\begin{array}{l}\text { F 10-13 HIPS } 1-8 \text { m; F } \\
\text { 10-13 blank HIPS }\end{array}$ & 14.67 & $\begin{array}{c}204.0934 \\
203.0854 \\
101.0387\end{array}$ & $\begin{array}{c}\mathrm{C}_{16} \mathrm{H}_{12} \\
\mathrm{C}_{16} \mathrm{H}_{11} \\
\mathrm{C}_{8} \mathrm{H}_{5}\end{array}$ & $\begin{array}{c}0.22 \\
-0.57 \\
1.17\end{array}$ & & 4 \\
\hline $\begin{array}{l}\text { F 9-15 HIPS 1-8 m; F 9-15 } \\
\text { blank HIPS }\end{array}$ & 23.7 & $\begin{array}{c}312.18782 \\
97.1639 \\
206.1091 \\
129.0698 \\
91.0543\end{array}$ & $\begin{array}{c}\mathrm{C}_{24} \mathrm{H}_{24} \\
\mathrm{C}_{23} \mathrm{H}_{21} \\
\mathrm{C}_{16} \mathrm{H}_{14} \\
\mathrm{C}_{10} \mathrm{H}_{9} \\
\mathrm{C}_{7} \mathrm{H}_{7}\end{array}$ & $\begin{array}{c}N D \\
0.43 \\
0.38 \\
-0.23 \\
0.60\end{array}$ & 1e-Phenyl-4e-(1-phenylethyl)-tetralin & 2 b) \\
\hline
\end{tabular}

The $m / z$ is given in descending order from molecular ion to detected fragment ions.

*Identification confidence degree as proposed by Schymanski et al. (2014); ND, non-detected.

inducers of the AhR pathway over time compared to more readily degradable compounds, such as PAHs (Lee et al., 2013; Larsson et al., 2014). Therefore, AhR agonists in plastic extracts were probably not PCDD/Fs and coplanar PCBs. It is possible that the sulfuric acid reaction time of $3 \mathrm{~h}$ used in this study was not sufficient to completely break down acid labile compounds, like PAHs. A previous study demonstrated that a sulfuric acid treatment of $1 \mathrm{~h}$ was able to reduce the AhR-mediated activity of a mixture of the 16 EPA PAHs by $50 \%$, while no response was induced after $10 \mathrm{~h}$ of acid treatment of the mixture (Villeneuve et al., 2002). Consequently, acid-degradable compounds, such as PAHs, could still have been present in the sample extracts. Since Heron Island is also used for recreational purposes, it is likely that PAHs were emitted into the water by boat traffic and sorbed to the plastic pellets.

LDPE, HDPE, and HIPS samples had similar bio-TEQs for the different deployment times. Similar sorption capacities have been reported before for PAHs to plastic pellets of these polymers (Rochman et al., 2013b), and for PCBs for PE polymer types (Rochman et al., 2013a). Sorption of other compound classes to LDPE and HDPE is therefore likely to be similar as well, due to the similar polymer structure.

The observed cytotoxicity in deployed HIPS pellets and in the HIPS polymer blank indicates that the cause of the cytotoxicity originated from the pristine pellet itself. Indeed, the styrene monomer is known to be disruptive and carcinogenic to the endocrine system (Ohyama et al., 2001; Lithner et al., 2011), and some styrene dimers and trimers have been reported to act as weak AhR agonists (Hong et al., 2016). However, cytotoxic effects of polystyrene (PS) monomers or oligomers have not been reported in H4IIepGudluc1.1cells, to the best of our knowledge, although hepatotoxicity of styrene and styrene oxide has been reported in mice (Gadberry et al., 1996).

Due to the relative remote area of the deployment site in the present study a rather small anthropogenic impact was expected. Therefore, the obtained bio-TEQs might act as benchmarks of control sites for AhR-mediated potencies in similar studies. In the previous study by Schönlau et al. (2019) the greatest AhR-mediated potency (277 pg/g) was observed in extracts of LDPE pellets, which had been deployed for 9 months in an urban harbor with industrial and recreational activity. The greatest AhR-mediated potency in the current study for LDPE was almost five times less $(57 \mathrm{pg} / \mathrm{g}$ LDPE I $6 \mathrm{~m}$ ) and almost three times less in HDPE (100 pg/g HDPE I $8 \mathrm{~m}$ ). The difference in AhR-mediated activities between the two study sites is quite small considering the fact of the two very different locations and that in the previous study no acid treatment was conducted. The herein analyzed plastic pellets were only deployed for 8 months and a longer deployment time at the present study location could lead to greater AhRmediated potencies because elevated levels of especially octachlorinated dibenzo-p-dioxins have been reported in sediment and seagrass samples from the Great Barrier reef (McLachlan et al., 2001). In general higher substituted congeners need longer times to sorb to microplastics, as sorption kinetics decrease with increasing hydrophobicity (Endo et al., 2013). It has been suggested that marine microplastics will be at equilibrium with most HOCs by 2 years (Koelmans et al., 2016). However, it has also been demonstrated that AhR-mediated potencies in 12 months deployed plastic pellets were lower compared to 9 months deployed plastic pellets (Schönlau et al., 2019). This suggests that there is not a steady state of compounds sorbed to marine microplastics and peak concentrations might be reached much earlier than expected and/or vary throughout the residence time in the marine system.

Although observed AhR-mediated potencies were small in the analyzed samples, for environmental implications it is, however, noteworthy that pristine pellets of HDPE and the fractionated HIPS elicited a measurable AhR-mediated response before deployment. The unfractionated blank sample of HIPS even led to cytotoxicity in the bioassay. These results suggest that the polymers themselves have a potential to 
activate the AhR-mediated pathway, thus might be of greater concern than other polymers regarding an exposure in the marine environment.

\section{Occurrence of HOCs on Deployed Plastics}

Because PE has been widely used in passive sampling devices (Zabiegala et al., 2010), sorption of compounds to LDPE and HDPE pellets was expected. In general, relatively low levels of PCBs, organochlorine pesticides and PAHs have been reported in sediment and surface water samples from the Great Barrier Reef, with the exception of sites that are close to human activity, such as harbors and sites adjacent to agriculture (Haynes and Johnson, 2000; Haynes et al., 2000). In fact, anthropogenic impact via agricultural and industrial runoff were the major sources of contamination (Haynes and Johnson, 2000; Packett et al., 2009). Therefore, the deployed plastic pellets were expected to contain low concentrations of such HOCs. This was reflected by the relatively small concentrations of analyzed target compounds in deployed samples. The predominance of hexa-chlorinated PCB congeners is similar to patterns observed for beached plastic particles of PE (Hirai et al., 2011). Due to the lower recoveries of PCB\# 138 (66\%) and PCB \#153 (42\%) in LDPE pellets, which were observed in the spiked recovery test (see Table S2), it indicates that the herein reported values for both congeners are underestimations. While PBDEs can be expected to be present already in the pristine pellets as additives (Hirai et al., 2011), it was unexpected to find PCBs in measurable concentrations in pristine plastic pellets, and has not been reported before. For HDPE pellets it was notable that the total PCB concentration increased after 1 month of deployment compared to the blank level, and then declined in concentration with time of deployment. It is unclear whether this was due to desorption into the water phase after an initial adsorption or absorption into the polymer matrix. In deployed LDPE samples concentrations of the predominant congeners PCB \#138 and PCB \#153 suggest a sorption from the surrounding environment to the pellets because the congeners were not detected in the blank polymer.

The predominance of the PBDE congener \#47 in deployed samples is not surprising because this congener is commonly detected in seawater (Mizukawa et al., 2009; Wang et al., 2011). In deployed LDPE samples concentrations of BDE47 were higher compared to the measured concentration in the blank polymer which suggests a sorption from seawater. However, in deployed HDPE samples the concentrations were slightly lower than in the blank polymer which suggest that no BDE47 sorbed from the surrounding water to the pellets. The spiked recovery of BDE47 from LDPE pellets was $43 \%$ (Table S2) which indicates that measured values of BDE47 in LDPE samples were underestimated.

Overall, while the biological activities showed an increase over the time of deployment, this could not be seen in total concentrations of detected HOCs in all samples. LDPE samples expressed a fluctuation of total concentrations of analyzed compounds during the time of deployment with a final increase and HDPE samples showed firstly an increase followed by a decrease with a rather constant concentration at all three subsequent sampling dates. The average water temperature during the time of deployment was reported to decrease from $27^{\circ} \mathrm{C}$ at the time of deployment to $20^{\circ} \mathrm{C}$ in August, and increased again up to $22^{\circ} \mathrm{C}$ in October when the last samples were taken (http://weather.aims.gov.au/\#/station/130). Because the water temperature does not reflect the observed patterns in bioactivities and measured chemicals over the time of deployment, it suggest that the water temperature did not have a significant influence on the uptake of AhR agonist nor analyzed HOCs. However, the longer the plastic pellets were deployed in the water, the more have they been impacted by weathering. The degree of weathering has been reported to be positively correlated with increasing concentrations of PCBs (Endo et al., 2005), therefore concentrations of other compounds might also be positively correlated with more weathered plastic pellets which was only observed in the increasing bio-TEQs over time.

The calculated chem-TEQ values demonstrated a negligible contribution of the quantified PCBs and OCDD to the total induced AhR-mediated activities $(<0.001 \%)$ even though concentrations below LOQ were also considered. Although the occurrence of several halogenated natural compounds produced by algae, sponges and other marine biota in the Great Barrier Reef is well-known (Vetter et al., 2018), they were not likely to be the inducers of the observed AhRmediated responses. Halogenated dimethyl bipyrroles (HDBPs), for example, have been detected as AhR agonists, but they are relatively persistent and even elicited greater AhR-mediated potencies following $48 \mathrm{~h}$ of exposure (Tittlemier et al., 2003). This would suggest that HDBPs also show an AhR-mediated activity following a $72 \mathrm{~h}$ exposure and herein analyzed plastic extracts did not elicit an AhR-mediated response following a $72 \mathrm{~h}$ exposure.

In the present study we used a quick and more exhaustive extraction technique compared to, for example, leaching, to screen for total concentrations of AhR-active compounds in marine microplastics. This method does not reflect the bioavailable fraction of the compounds, but if bio-TEQs of total extracts are already low, it might not be necessary to further investigate the bioavailability of the compounds causing the activity since the bioavailable fraction can be expected to be even lower. The calculated bio-TEQs in the present study were smaller than previously measured bio-TEQs in LDPE plastic pellets that were deployed in an urban marine harbor (Schönlau et al., 2019). Furthermore, since no measurable AhRmediated activities were induced following a $72 \mathrm{~h}$ exposure, compounds in the present study were readily metabolized, thus probably present a smaller risk to organisms. It has been hypothesized that the contribution of ingested microplastics in transferring HOCs to organisms is rather negligible and might be much greater from other marine sources, such as colloids and dissolved organic carbon, because they hold greater amounts of sorbed HOCs (Koelmans et al., 2016). In addition, the dietary intake of HOCs via prey is likely a more important exposure pathway for many marine organisms compared to microplastics, and has so far been shown in northern fulmars (Herzke et al., 2016). However, the exposure to microplastics will most certainly vary according to different marine habitats, 
and accumulation zones or point sources of microplastics might be more important sites for the exposure to plasticderived HOCs.

\section{Performance of the GC-MS Fractionation Platform}

By chemical analysis of the fractionated standard mixture with a HRMS system, $88 \%$ of the compounds in the mixture were detected. The calculated recoveries were, however, small for the majority of compounds. For identification of bioactive compounds the small recoveries might not demonstrate a problem as long as the concentration of a compound in the fraction is sufficient for obtaining a measurable signal in the bioassay and chemical analysis. But for the assessment of the contribution of a compound to the overall potency in the bioassay a quantification of the compound is necessary. For more certainty of the calculated recoveries it would be beneficial to spike the fractions with labeled internal standards which also accounts for matrix effects. Nevertheless, for further studies it needs to be investigated if a loss of compounds already occurs in the fractionation step or if the subsequent transfer and evaporation of the eluate in fractionation vials leads to a loss of compounds. The high recoveries of lower molecular weight PAHs need to be further investigated, but are likely due to background contamination during fractionation since the compounds are present in air, especially naphthalene (Buckpitt et al., 2010; Jia and Batterman, 2010), and colleting fractions from 15 injections leaves time for airborne contamination.

Among the analyzed pesticides only methoxychlor and p, p'DDT have been reported to be weak AhR agonists (Takeuchi et al., 2008), but methoxychlor could not be identified in fractions of the known standard mixture.

However, the implications for the present study can be expected to be negligible because high recoveries of low molecular weight PAHs would not contribute to AhR-mediated activities in the samples since they are not AhR agonists. Also non-fractionated total plastic extracts were investigated qualitatively for target, suspect, and non-target chemicals and results were compared with results of fractionated samples. The same compounds were found in total and fractionated extracts in similar intensities.

\section{Bioassay Testing of Fractionated Pooled Plastic Extracts}

Bio-TEQs derived from individual plastic pellets were greater compared to bio-TEQs obtained by pooled samples. This was not expected and could implicate that concentrations of AhR agonists in pooled samples were not high enough for identification, even though collecting 15 repeated injections.

That total pooled extracts demonstrated greater bioactivities than fractionated samples be explained by higher tested plastic concentrations of total extracts compared to plastic concentrations in the individual fractions. The amount of plastic that was tested in the bioassay in fractions of HDPE 1-8 $\mathrm{m}$ was about $50 \%$ of the amount of tested plastic in the total extract of HDPE 1-8 $\mathrm{m}$, and in fractions of LDPE 1-8 $\mathrm{m}$ the amount of plastic was around $40 \%$ of the amount of the total extract of LDPE 1-8 m. This means that the concentration of some agonists in the fractions could have been too low to even reach $\mathrm{EC}_{10}$ levels, thus did not contribute to the calculated bio-TEQs. Furthermore, total extracts integrate the entire bioactivity of compounds present in the mixture. This means that AhR agonists present in the mixture can act, for instance, in a synergistic or additive manner. Separated in fractions, AhR agonists will only elicit AhR-mediated responses based on their potencies and concentrations in each fraction. For instance, weak AhR agonists that did not induce a measurable bioactivity in single fractions could have added up to the bioactivity in the total extracts. The AhR-mediated potencies of fractions 12 and 14 added up together induced 54 and $38 \%$ of the potency measured in total extracts of HDPE 1-8 $\mathrm{m}$ and LDPE 1-8 $\mathrm{m}$, respectively. Additionally, antagonists might have been present in the extracts which can possibly bind easier to the AhR in individual fractions due to less competition with AhR agonists, thus decreasing the bioactivity in fractions.

Overall, the GC-MS fractionation platform was efficient in reducing the complexity and isolating the bioactive drivers in the samples. This was especially noted for HIPS for which only one fraction showed cytotoxicity. The detected bioactivities in later eluting fractions (fraction 12 and 14) indicate that the AhRmediated activities in extracts of plastic samples were caused by more hydrophobic compounds. Since the AhR-mediated activities were observed in the same fractions, regardless of the type of polymer, it is most likely the same compounds that were causing the bioactivities.

\section{Chemical Analysis of Fractionated Pooled Plastic Extracts by HRMS}

The conducted target analysis of fractionated pooled plastic samples revealed a few PAHs in earlier fractions (fraction 2-6). Because all of the detected PAHs have been reported to be present in polystyrene pristine pellets (Rochman et al., 2013b), and all PAHs, except for fluorene, had similar intensities in the TICs of deployed HIPS compared to blank HIPS, the PAHs were most likely present from the manufacturing of the HIPS pellets. Of all the detected PAHs only pyrene is a weak AhR agonist, while the others have not been observed to act as AhR agonists (Larsson et al., 2012). Pyrene was detected in fraction 5 of HIPS $1-8 \mathrm{~m}$ and in blank HIPS, and not in a fraction which demonstrated a measurable AhR activity.

The tentatively identified compound 1e-Phenyl-4e-(1phenylethyl)-tetralin, in fractions 9 to15 of pooled HIPS and blank HIPS, was found to be a styrene trimer of polystyrene and has been detected in extracts of sediments as residues of PS contamination (Hong et al., 2016), as well as in food packaging materials (Choi et al., 2005; Nakai et al., 2014). Some styrene trimers have been reported as weak AhR agonists (Hong et al., 2016), and it is possible that 1e-Phenyl-4e-(1phenylethyl)-tetralin contributed to the AhR-mediated response in fractions 12 and 14 of pooled HIPS, and fraction 12 of blank HIPS. However, it is unclear which isomer of 1e-Phenyl-4e-(1phenylethyl)-tetralin was present in the herein analyzed HIPS 
fractions. A cytotoxicity of 1e-Phenyl-4e-(1-phenylethyl)-tetralin has not been reported in the literature. The occurrence of this compound in the blank, as well as in the deployed samples, corroborates the hypothesis that the plastic polymer itself was the source of this compound.

Furthermore, hexamethylbenzene was detected in fractions of deployed and blank HIPS, which suggests that also this compound was originating from the HIPS pellets itself. Various substituted benzenes have been identified in PS food packaging material (Nerin et al., 1998), but no AhR-mediated potency of hexamethylbenzene was found in the literature.

Compounds specific for the bioactive fractions 12 and 14 of HDPE 1-8 $\mathrm{m}$ and LDPE 1-8 $\mathrm{m}$ could not be identified using the conducted effect-directed approach. This was probably due to low concentrations of compounds in the fractions and a greater number of repeated injections would have been necessary to enable a detection. In addition, the bioactive compounds might not form stable ions in the used electron impact ionization of the mass spectrometer and inhibit a detection.

However, AhR-mediated potencies of individual plastic pellets compared to AhR-mediated potencies of blank polymers indicated that $A h R$ agonists were sorbed from the marine environment during the time of deployment (Table S6 and Figure 1), and the used DR CALUX ${ }^{\circledR}$ assay was observed to be a more sensitive tool in the detection of AhR agonists compared to chemical analysis.

\section{CONCLUSIONS}

Bio-TEQs in the present study were similar for LDPE, HDPE, and HIPS pellets and most likely reflect background levels of AhR-mediated activities from the marine environment, because of the supposed small anthropogenic impact at the deployment site reflected in the low bio-TEQs of the deployed plastics. HIPS might be of greater concern for the environment due to the observed cytotoxicity of the pristine pellet. The contribution of PCB 105, 118, 156, 157 and OCDD to the measured bioactivities was negligible $(<0.001 \%)$, therefore indicating the presence of other AhR agonists. However, the conducted effectdirected approach was not successful in identifying the bioactive compounds in deployed LDPE and HDPE samples. First and foremost, the concentrations of AhR agonist in the samples were small, as indicated by low bio-TEQs of pooled plastics, and likely below detection limits of the used instrumentation. The used GCMS fractionation platform was, however, successful in separating 118 out of 134 HOCs, but recoveries for most compounds were low and present a difficulty for samples with low levels of pollution. The fractionation set up reduced the complexity of the samples, and only three fractions out of 17 showed an

\section{REFERENCES}

Bjurlid, F., Roos, A., Ericson Jogsten, I., and Hagberg, J. (2018). Temporal trends of $\mathrm{pbdd} / \mathrm{fs}, \mathrm{pcdd} / \mathrm{fs}$, pbdes and pcbs in ringed seals from the baltic sea (pusa hispida botnica) between 1974 and 2015. Sci. Total Environ. 616-617:1374-1383. doi: 10.1016/j.scitotenv.2017.10.178
AhR-mediated activity. 1e-Phenyl-4e-(1-phenylethyl)-tetralin, was discovered by non-target analysis in fractions of both deployed HIPS and blank HIPS, indicating the compound originated from the pellets itself. This compound is suspected to having contributed to the observed AhR-mediated potencies in fraction 12 and 14 of HIPS samples as it has been reported to be an AhR agonist. Overall, the bioassay presented to be a sensitive tool in the detection of AhR agonist sorbed to marine microplastics and demonstrates the importance of bioassay analysis along with chemical analysis in order to estimate risks of sorbed compounds.

\section{DATA AVAILABILITY}

The datasets for this manuscript are not publicly available because of the size of the data set, especially the non-target data. Requests to access the datasets should be directed to Christine Schönlau [Christine.Schonlau@oru.se].

\section{AUTHOR CONTRIBUTIONS}

$\mathrm{AR}, \mathrm{CS}, \mathrm{ML}$, and AK designed the study. AR and $\mathrm{RvdZ}$ carried out the deployment and sampling of microplastics. CS carried out the laboratory work including target chemical and bioassay analysis. FD did the non-target and suspect chemical analysis. CS and FD carried out the data analysis. CS wrote the manuscript and ML, FD, ME, AR, and $\mathrm{AK}$ contributed to the manuscript by commenting on the manuscript.

\section{FUNDING}

This study was funded by the Swedish Research Council for Environment, Agricultural Sciences and Spatial Planning (2232014-1064) and by the EnForce research project (20160019), funded by the Knowledge foundation.

\section{ACKNOWLEDGMENTS}

CS would like to thank Jochen Mueller for use of laboratory facilities at the Queensland Alliance for Environmental Health Sciences, Brisbane, Australia, and Rebecca Bülow for help with the bioassay testing.

\section{SUPPLEMENTARY MATERIAL}

The Supplementary Material for this article can be found online at: https://www.frontiersin.org/articles/10.3389/fenvs. 2019.00120/full\#supplementary-material

Buckpitt, A., Kephalopoulos, S., Koistinen, K., Kotzias, D., Morawska, L., and Sagunski, H. (2010). "Who guidelines for indoor air quality: selected pollutants," in Who Guidelines for Indoor Air Quality: Selected Pollutants.

Choi, J. O., Jitsunari, F., Asakawa, F., and Lee, D. (2005). Migration of styrene monomer, dimers and trimers from polystyrene to food simulants. Food Addit. Contaminants 22, 693-699. doi: 10.1080/02652030500160050 
Denison, M. S., and Heath-Pagliuso, S. (1998). The ah receptor: a regulator of the biochemical and toxicological actions of structurally diverse chemicals. $B$ Environ. Contam. Tox 61, 557-568. doi: 10.1007/PL00002973

Endo, S., Takizawa, R., Okuda, K., Takada, H., Chiba, K., Kanehiro, H., et al. (2005). Concentration of polychlorinated biphenyls (pcbs) in beached resin pellets: variability among individual particles and regional differences. Mar. Pollut. Bull. 50, 1103-1114. doi: 10.1016/j.marpolbul.2005.04.030

Endo, S., Yuyama, M., and Takada, H. (2013). Desorption kinetics of hydrophobic organic contaminants from marine plastic pellets. Mar. Pollut. Bull. 74, 125-131. doi: 10.1016/j.marpolbul.2013.07.018

Gadberry, M. G., DeNicola, D. B., and Carlson, G. P. (1996). Pneumotoxicity and hepatotoxicity of styrene and styrene oxide. J. Toxicol. Env. Health 48, 273-294. doi: 10.1080/009841096161339

Grung, M., Naes, K., Fogelberg, O., Nilsen, A. J., Brack, W., Lubckevon Varel, U., et al. (2011). Effects-directed analysis of sediments from polluted marine sites in norway. J. Toxicol. Env. Heal. A 74, 439-454. doi: $10.1080 / 15287394.2011 .550555$

Haynes, D., and Johnson, J. E. (2000). Organochlorine, heavy metal and polyaromatic hydrocarbon pollutant concentrations in the great barrier reef (australia) environment: a review. Mar. Pollut. Bull. 41, 267-278. doi: 10.1016/S0025-326X(00)00134-X

Haynes, D., Müller, J., and Carter, S. (2000). Pesticide and herbicide residues in sediments and seagrasses from the great barrier reef world heritage area and queensland coast. Mar. Pollut. Bull 41, 279-287. doi: $10.1016 / \mathrm{S} 0025-326 \mathrm{X}(00) 00097-7$

Herzke, D., Anker-Nilssen, T., Nost, T. H., Gotsch, A., Christensen-Dalsgaard, S., Langset, M., et al. (2016). Negligible impact of ingested microplastics on tissue concentrations of persistent organic pollutants in northern fulmars off coastal norway. Environ. Sci. Technol. 50, 1924-1933. doi: 10.1021/acs.est.5b04663

Hirai, H., Takada, H., Ogata, Y., Yamashita, R., Mizukawa, K., Saha, M., et al. (2011). Organic micropollutants in marine plastics debris from the open ocean and remote and urban beaches. Mar. Pollut. Bull 62, 1683-1692. doi: 10.1016/j.marpolbul.2011.06.004

Hong, S., Lee, J., Lee, C., Yoon, S. J., Jeon, S., Kwon, B.-O., et al. (2016). Are styrene oligomers in coastal sediments of an industrial area aryl hydrocarbon-receptor agonists? Environ. Pollut. 213, 913-921. doi: 10.1016/j.envpol.2016.03.025

Jia, C., and Batterman, S. (2010). A critical review of naphthalene sources and exposures relevant to indoor and outdoor air. Int. J. Environ. Res. Public Health 7, 2903-2939. doi: 10.3390/ijerph7072903

Jonker, W., Clarijs, B., de Witte, S. L., van Velzen, M., de Koning, S., Schaap, J., et al. (2016). Gas chromatography fractionation platform featuring parallel flameionization detection and continuous high-resolution analyte collection in 384well plates. J. Chromatogr. A 1462, 100-106. doi: 10.1016/j.chroma.2016.07.068

Jonker, W., Zwart, N., Stockl, J. B., de Koning, S., Schaap, J., Lamoree, M. H., et al. (2017). Continuous fraction collection of gas chromatographic separations with parallel mass spectrometric detection applied to cell-based bioactivity analysis. Talanta 168, 162-167. doi: 10.1016/j.talanta.2017.02.067

Koelmans, A. A., Bakir, A., Burton, G. A., and Janssen, C. R. (2016). Microplastic as a vector for chemicals in the aquatic environment: critical review and model-supported reinterpretation of empirical studies. Environ. Sci. Technol. 50, 3315-3326. doi: 10.1021/acs.est.5b06069

Larsson, M., Hagberg, J., Giesy, J. P., and Engwall, M. (2014). Time-dependent relative potency factors for polycyclic aromatic hydrocarbons and their derivatives in the h4iie-luc bioassay. Environ. Toxicol. Chem. 33, 943-953. doi: 10.1002/etc. 2517

Larsson, M., Hagberg, J., Rotander, A., van Bavel, B., and Engwall, M. (2013). Chemical and bioanalytical characterisation of pahs in risk assessment of remediated pah-contaminated soils. Environ. Sci. Pollut. Res. 20, 8511-8520. doi: 10.1007/s11356-013-1787-6

Larsson, M., Orbe, D., and Engwall, M. (2012). Exposure time-dependent effects on the relative potencies and additivity of pahs in the ah receptor-based h4iie-luc bioassay. Environ. Toxicol. Chem. 31, 1149-1157. doi: 10.1002/etc.1776

Lee, K. T., Hong, S., Lee, J. S., Chung, K. H., Hilscherova, K., Giesy, J. P., et al. (2013). Revised relative potency values for pcdds, pcdfs, and non-ortho-substituted pcbs for the optimized h4iie-luc in vitro bioassay. Environ. Sci. Pollut. Res. 20, 8590-8599. doi: 10.1007/s11356-013$1770-2$
Lithner, D., Larsson, A., and Dave, G. (2011). Environmental and health hazard ranking and assessment of plastic polymers based on chemical composition. Sci. Total Environ. 409, 3309-3324. doi: 10.1016/j.scitotenv.2011.04.038

Lubcke-von Varel, U., Streck, G., and Brack, W. (2008). Automated fractionation procedure for polycyclic aromatic compounds in sediment extracts on three coupled normal-phase high-performance liquid chromatography columns. J. Chromatogr. A 1185, 31-42. doi: 10.1016/j.chroma.2008.01.055

Marlowe, J. L., and Puga, A. (2005). Aryl hydrocarbon receptor, cell cycle regulation, toxicity, and tumorigenesis. J. Cell. Biochem. 96, 1174-1184. doi: $10.1002 /$ jcb. 20656

Mato, Y., Isobe, T., Takada, H., Kanehiro, H., Ohtake, C., and Kaminuma, T. (2001). Plastic resin pellets as a transport medium for toxic chemicals in the marine environment. Environ. Sci. Technol. 35, 318-324. doi: $10.1021 /$ es 0010498

McLachlan, M. S., Haynes, D., and Müller, J. F. (2001). Pcdds in the water/sediment-seagrass-dugong (dugong dugon) food chain on the great barrier reef (australia). Environ. Pollut. 113, 129-134. doi: 10.1016/S0269-7491(00)00172-X

Mizukawa, K., Takada, H., Takeuchi, I., Ikemoto, T., Omori, K., and Tsuchiya, K. (2009). Bioconcentration and biomagnification of polybrominated diphenyl ethers (pbdes) through lower-trophic-level coastal marine food web. Mar. Pollut. Bull. 58, 1217-1224. doi: 10.1016/j.marpolbul.2009.03.008

Murk, A. J., Legler, J., Denison, M. S., Giesy, J. P., vandeGuchte, C., and Brouwer, A. (1996). Chemical-activated luciferase gene expression (calux): a novel in vitro bioassay for ah receptor active compounds in sediments and pore water. Fund. Appl. Toxicol. 33, 149-160. doi: 10.1093/toxsci/33.1.149

Nakai, M., Tsubokura, M., Suzuki, M., Fujishima, S., Watanabe, Y., Hasegawa, Y., et al. (2014). Genotoxicity of styrene oligomers extracted from polystyrene intended for use in contact with food. Toxicol. Rep. 1, 1175-1180. doi: $10.1016 /$ j.toxrep.2014.11.007

Nerin, C., Rubio, C., Cacho, J., and Salafranca, J. (1998). Parts-per-trillion determination of styrene in yoghurt by purge-and-trap gas chromatography with mass spectrometry detection. Food Additives Contaminants 15, 346-354. doi: $10.1080 / 02652039809374650$

Ohyama, K. I., Nagai, F., and Tsuchiya, Y. (2001). Certain styrene oligomers have proliferative activity on mcf-7 human breast tumor cells and binding affinity for human estrogen receptor $\alpha$. Environ. Health Persp. 109, 699-703. doi: 10.1289/ehp.01109699

Packett, R., Dougall, C., Rohde, K., and Noble, R. (2009). Agricultural lands are hot-spots for annual runoff polluting the southern great barrier reef lagoon. Mar. Pollut. Bull. 58, 976-986. doi: 10.1016/j.marpolbul.2009.02.017

Pieke, E., Heus, F., Kamstra, J. H., Mladic, M., van Velzen, M., Kamminga, D., et al. (2013). High-resolution fractionation after gas chromatography for effect-directed analysis. Anal. Chem. 85, 8204-8211. doi: 10.1021/ac401384q

Poster, D. L., Schantz, M. M., Sander, L. C., and Wise, S. A. (2006). Analysis of polycyclic aromatic hydrocarbons (pahs) in environmental samples: a critical review of gas chromatographic (gc) methods. Anal. Bioanal. Chem. 386, 859-881. doi: 10.1007/s00216-006-0771-0

Rios, L. M., Moore, C., and Jones, P. R. (2007). Persistent organic pollutants carried by synthetic polymers in the ocean environment. Mar. Pollut. Bull. 54, 1230-1237. doi: 10.1016/j.marpolbul.2007.03.022

Rochman, C. M., Hoh, E., Hentschel, B. T., and Kaye, S. (2013a). Long-term field measurement of sorption of organic contaminants to five types of plastic pellets: implications for plastic marine debris. Environ. Sci. Technol. 47, 1646-1654. doi: $10.1021 /$ es303700s

Rochman, C. M., Manzano, C., Hentschel, B. T., Simonich, S. L., and Hoh, E. (2013b). Polystyrene plastic: a source and sink for polycyclic aromatic hydrocarbons in the marine environment. Environ. Sci. Technol. 47, 13976-13984. doi: 10.1021/es403605f

Schönlau, C., Larsson, M., Lam, M. M., Engwall, M., Giesy, J. P., Rochman, C., et al. (2019). Aryl hydrocarbon receptor-mediated potencies in field-deployed plastics vary by type of polymer. Environ. Sci. Pollut. Res. 26, 9079-9088. doi: 10.1007/s11356-019-04281-4

Schymanski, E. L., Jeon, J., Gulde, R., Fenner, K., Ruff, M., Singer, H. P., et al. (2014). Identifying small molecules via high resolution mass spectrometry: communicating confidence. Environ. Sci. Technol. 48, 2097-2098. doi: 10.1021/es5002105 
Takeuchi, S., Iida, M., Yabushita, H., Matsuda, T., and Kojima, H. (2008). In vitro screening for aryl hydrocarbon receptor agonistic activity in 200 pesticides using a highly sensitive reporter cell line, dr-ecoscreen cells, and in vivo mouse liver cytochrome p450-1a induction by propanil, diuron and linuron. Chemosphere 74, 155-165. doi: 10.1016/j.chemosphere.2008.08.015

Teuten, E. L., Saquing, J. M., Knappe, D. R., Barlaz, M. A., Jonsson, S., Bjorn, A., et al. (2009). Transport and release of chemicals from plastics to the environment and to wildlife. Philos. T. R. Soc. B 364, 2027-2045. doi: $10.1098 /$ rstb. 2008.0284

Tittlemier, S. A., Kennedy, S. W., Hahn, M. E., Reddy, C. M., and Norstrom, R. J. (2003). Naturally produced halogenated dimethyl bipyrroles bind to the aryl hydrocarbon receptor and induce cytochrome p4501a and porphyrin accumulation in chicken embryo hepatocytes. Environ. Toxicol. Chem. 22, 1622-1631. doi: 10.1002/etc.5620220726

Tombesi, N., Pozo, K., Alvarez, M., Pribylova, P., Kukucka, P., Audy, O., et al. (2017). Tracking polychlorinated biphenyls (pcbs) and polybrominated diphenyl ethers (pbdes) in sediments and soils from the southwest of buenos aires province, argentina (south eastern part of the grulac region). Sci. Total. Environ. 575, 1470-1476. doi: 10.1016/j.scitotenv.2016.10.013

USEPA (2014). Method 4435-Screening for Dioxin-Like Chemical Activity in Soil and Sediments Using the Calux $($ Bioassay and Teq Determinations. Available online at: https://www.epa.gov/sites/production/files/2015-12/documents/ 4435.pdf

Van, A., Rochman, C. M., Flores, E. M., Hill, K. L., Vargas, E., Vargas, S. A., et al. (2012). Persistent organic pollutants in plastic marine debris found on beaches in san diego, california. Chemosphere 86, 258-263. doi: 10.1016/j.chemosphere.2011.09.039

Vetter, W., Kaserzon, S., Gallen, C., Knoll, S., Gallen, M., Hauler, C., et al. (2018). Occurrence and concentrations of halogenated natural products derived from seven years of passive water sampling (2007-2013) at normanby island, great barrier reef, australia. Mar. Pollut. Bull. 137, 81-90. doi: 10.1016/j.marpolbul.2018.09.032
Villeneuve, D. L., Blankenship, A. L., and Giesy, J. P. (2000). Derivation and application of relative potency estimates based on in vitro bioassay results. Environ. Toxicol. Chem. 19, 2835-2843. doi: 10.1002/etc.56201 91131

Villeneuve, D. L., Khim, J. S., Kannan, K., and Giesy, J. P. (2002). Relative potencies of individual polycyclic aromatic hydrocarbons to induce dioxinlike and estrogenic responses in three cell lines. Environ. Toxicol. 17, 128-137. doi: 10.1002/tox.10041

Wang, J., Lin, Z., Lin, K., Wang, C., Zhang, W., Cui, C., et al. (2011). Polybrominated diphenyl ethers in water, sediment, soil, and biological samples from different industrial areas in zhejiang, china. J. Hazard. Mater. 197, 211-219. doi: 10.1016/j.jhazmat.2011.09.078

Weiss, J. M., Hamers, T., Thomas, K. V., van der Linden, S., Leonards, P. E., and Lamoree, M. H. (2009). Masking effect of anti-androgens on androgenic activity in european river sediment unveiled by effect-directed analysis. Anal. Bioanal. Chem. 394, 1385-1397. doi: 10.1007/s00216-009-2807-8

Zabiegala, B., Kot-Wasik, A., Urbanowicz, M., and Namiesnik, J. (2010). Passive sampling as a tool for obtaining reliable analytical information in environmental quality monitoring. Anal. Bioanal. Chem. 396, 273-296. doi: 10.1007/s00216-009-3244-4

Conflict of Interest Statement: The authors declare that the research was conducted in the absence of any commercial or financial relationships that could be construed as a potential conflict of interest.

Copyright (c) 2019 Schönlau, Larsson, Dubocq, Rotander, van der Zande, Engwall and Kärman. This is an open-access article distributed under the terms of the Creative Commons Attribution License (CC BY). The use, distribution or reproduction in other forums is permitted, provided the original author(s) and the copyright owner(s) are credited and that the original publication in this journal is cited, in accordance with accepted academic practice. No use, distribution or reproduction is permitted which does not comply with these terms. 